\title{
TWO NONEXISTENCE THEOREMS ON PARTITIONS
}

\author{
D. H. LEHMER
}

The theory of partitions contains a number of theorems which assert that the number of partitions of a given number into parts subjected to a certain restriction is the same as the number of partitions restricted in some other way. A common type of restricted partition is one in which all parts are distinct. We have for example the famous theorem of Euler $^{1}$ (1748):

EULER'S THEOREM. The number of partitions of $n$ into distinct parts is the same as the number of partitions of $n$ into odd parts.

The notion of distinctness of parts may be altered in two directions. One may relax it to some extent and admit partitions in which no part is repeated more than a given number of times. In this case we have the beautiful theorem of Glaisher ${ }^{2}$ (1883).

GLAISHER'S THEOREM. The number of partitions of $n$ in which no part is repeated more than $r-1$ times is the same as the number of partitions of $n$ into parts not divisible by $r$.

This theorem obviously becomes Euler's theorem when $r=2$.

On the other hand the notion of distinctness may be further restricted so as to include only those partitions in which the parts differ by $d$ or more. For $d=0$, we have completely unrestricted partitions. For $d=2$ we have a celebrated and difficult theorem discovered independently by Rogers ${ }^{3}$ (1894), Schur $^{4}$ (1917) and Ramanujan ${ }^{5}$ (1919).

ROGERS' THEOREM. The number of partitions of $n$ into parts differing by 2 or more is the same as the number of partitions of $n$ into parts taken from the set $1,4,6,9,11,14,16, \cdots,(5 k+1,4), \cdots$.

Attempting to go further in this direction, Schur ${ }^{6}$ later (1926) proved the following theorem:

Presented to the Society, February 23, 1946; received by the editors December $15,1945$.

${ }^{1}$ L. Euler, Introductio analysin infinitorum, vol. 1, Lausanne, 1748, pp. 253-275.

2 J. W. L. Glaisher, Messenger of Mathematics vol. 12 (1883) pp. 158-170.

${ }^{3}$ L. J. Rogers, Proc. London Math. Soc. (1) vol. 25 (1894) pp. 328-329.

4 I. Schur, Akademie der Wissenschaften, Berlin, Sitzungsberichte (1917) pp. 302321.

- S. Ramanujan, Proc. Cambridge Philos. Soc. vol. 19 (1919) pp. 211-216.

- I. Schur, Akademie der Wissenschaften, Berlin, Sitzungsberichte (1926) pp. 488495. See also W. Gleissberg, Math. Zeit. vol. 28 (1928) pp. 372-382. 
SCHUR'S THEOREM. The number of partitions of $n$ into parts which differ by 3 or more, and such that parts divisible by 3 differ by at least 6 , is the same as the number of partitions of $n$ into parts taken from the set $1,7,13,19,25,31,37, \cdots,(6 k+1), \cdots$.

The above theorems have corresponding analytical formulations. If we denote by $q_{d}(n)$ the number of partitions of $n$ into parts differing by $d$ or more, then Euler's and Rogers' theorems can be written:

$$
\begin{aligned}
\sum_{n=0}^{\infty} q_{1}(n) x^{n} & =\sum_{s=0}^{\infty} \frac{x^{s(s+1) / 2}}{(1-x)\left(1-x^{2}\right) \cdots\left(1-x^{s}\right)} \\
& =\prod_{\nu=1}^{\infty}\left(1-x^{2 \nu-1}\right)^{-1}=\prod_{\nu=1}^{\infty}\left(1+x^{\nu}\right)
\end{aligned}
$$

and

$$
\begin{aligned}
\sum_{n=0}^{\infty} q_{2}(n) x^{n} & =\sum_{s=0}^{\infty} \frac{x^{s^{2}}}{(1-x)\left(1-x^{2}\right) \cdots\left(1-x^{s}\right)} \\
& =\prod_{\nu=0}^{\infty}\left(1-x^{5 \nu+1}\right)^{-1}\left(1-x^{5 \nu+4}\right)^{-1} .
\end{aligned}
$$

As a matter of fact, (1) and (2) include rather more than is stated in the theorems, namely the facts that $q_{1}(n)$ and $q_{2}(n)$ are generated by the sums over $s$. They suggest also the well known ${ }^{7}$ case due to Euler in which $d=0$, so that $g_{0}(n)=p(n)$, the number of unrestricted partitions of $n$ :

(3) $\sum_{n=0}^{\infty} p(n) x^{n}=\sum_{s=0}^{\infty} \frac{x^{s}}{(1-x)\left(1-x^{2}\right) \cdots\left(1-x^{s}\right)}=\prod_{\nu=1}^{\infty}\left(1-x^{\nu}\right)^{-1}$.

The first parts of (1), (2) and (3) are special cases of a general generating identity

$$
\sum_{n=0}^{\infty} q_{d}(n) x^{n}=\sum_{s=0}^{\infty} \frac{x^{s+d s(s-1) / 2}}{(1-x)\left(1-x^{2}\right) \cdots\left(1-x^{s}\right)}
$$

which is proved in Theorem 1. As for the products in (1), (2), and (3), we show in Theorems 2 and 3 that no further examples exist; that is, that neither of the identities

$$
\sum q_{d}(n) x^{n}=\prod_{\nu=1}^{\infty}\left(1-x^{a_{v}}\right)^{-1} \quad(d \neq 0,1,2),
$$

7 See for instance Hardy and Wright, Introduction to the theory of numbers, Oxford, 1938, chap. 19, where also can be found proofs of Euler's and Rogers' theorems. 


$$
\sum q_{d}(n) x^{n}=\prod_{\nu=1}^{\infty}\left(1+x^{a_{\nu}}\right)
$$

can hold, no matter what set of distinct $a$ 's is chosen. In particular this means that no more theorems like those of Euler and Rogers are possible. In commenting on (2), P. A. MacMahon ${ }^{8}$ points out that $q_{2}(n)$ is also the number of partitions of $n$ in which each part is at least equal to the number of parts. For the general $d$ there exists a corresponding second interpretation of $q_{d}(n)$. If $d$ is even, $q_{d}(n)$ is the number of partitions of $n$ into parts greater than $(s-1) d / 2$, where $s$ is the number of parts. For $d$ odd, $q_{d}(n)$ is the number of partitions of $2 n$ into parts greater than $1+(s-1) d$ and having the same parity as $s-1$. The case $d=2$ is certainly the most elegant one. However for $d>0$ such sets of parts depend on $s$ and so such partitions are not of the kinds considered in Theorems 2 and 3.

THEOREM 1. Let $q_{d}(n)$ denote the number of partitions of $n$ into parts differing by $d$ or more; then

$$
\sum_{n=0}^{\infty} q_{d}(n) x^{n}=\sum_{s=0}^{\infty} \frac{x^{s+d_{s}(s-1) / 2}}{(1-x)\left(1-x^{2}\right) \cdots\left(1-x^{s}\right)} .
$$

Proof. The following graphical proof may be given. Any partition of the sort enumerated by $q_{d}(n)$ may be represented graphically by a series of rows of dots or "nodes," each row representing a part. Thus the following figure represents the case of the partition $15+10+5+2$ of 32 into parts differing by three or more.
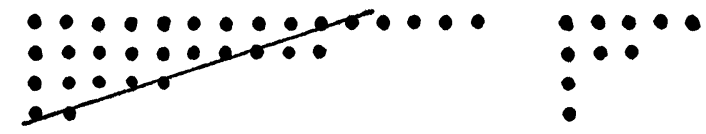

A line passing just below the last node in the first column having a slope of $1 / d$ divides the graph into a triangle or "head" and an irregular "tail." Graphs of this kind may be classified by the number of their rows or parts. It is clear that the number of nodes in the head of a graph of $s$ rows is the polygonal number

$$
s+d(s-1)+d(s-2)+\cdots+d=s+d(s-1) s / 2
$$

of order $d+2$. The $n-[s+d s(s-1) / 2]$ nodes in the tail may be translated to the right so as to form a "regular" graph which, when read by columns, gives a partition of $n-[s+d s(s-1) / 2]$ into parts not

' P. A. MacMahon, Combinatorial analysis, vol. 2, Cambridge, 1916. 
exceeding $s$. Conversely, one may start with a graph of such a partition and, by translating the $r$ th row $(r-1) d$ spaces to the left for $r=1,2, \cdots, s$ and affixing the head of $s$ rows, produce a graph of a partition of $n$ into parts differing by $d$ or more. Hence we may write

$$
q_{d}(n)=\sum_{s=0}^{\infty} p_{s}(n-s-d s(s-1) / 2)
$$

where $p_{s}(m)$ denotes the number of partitions of $m$ into parts not greater than $s, p_{0}(m)=0$ for $m>0, p_{s}(0)=1$. If we define $p_{s}(m)$ to be zero for $m<0$ we have, as is well known, ${ }^{7}$ the generating function

$$
\sum_{m=-\infty}^{\infty} p_{s}(m) x^{m}=\frac{1}{(1-x)\left(1-x^{2}\right) \cdots\left(1-x^{8}\right)} .
$$

Multiplying (5) by $x^{n}$ and summing over $n$ we have, in view of (6),

$$
\begin{aligned}
\sum_{n=0}^{\infty} q_{d}(n) x^{n} & =\sum_{s=0}^{\infty} x^{s+d s(s-1) / 2} \sum_{n=0}^{\infty} p_{s}(n-s-d s(s-1) / 2) x^{n-s-d s(s-1) / 2} \\
& =\sum_{s=0}^{\infty} \frac{x^{s+d s(s-1) / 2}}{(1-x)\left(1-x^{2}\right) \cdots\left(1-x^{s}\right)}
\end{aligned}
$$

which proves the theorem.

We now proceed to prove two nonexistence theorems.

THEOREM 2. The number $q_{d}(n)$ of partitions of $n$ into parts differing by $d$ or more is not equal to the number of partitions of $n$ into parts taken from any set $S$ of integers whatsoever, except when $d=0,1$, or 2 .

Remarks. The exceptional case $d=0$ is trivial since $S$ is in this case the set of all integers. For $d=1$, the set $S$ may be taken to be the set of all odd integers, giving us Euler's theorem. For $d=2$ the set $S$ consists of ali numbers of the form $5 x+1,4(x=0,1,2, \cdots)$, giving us Rogers' theorem. Hence we may suppose that $d \geqq 3$ in what follows.

Proof. Suppose that the theorem is false and that there exists such a set $S$ of integers $a_{1}<a_{2}<a_{3}<\ldots$ for $d \geqq 3$; then by Theorem 1 we would have

$$
\begin{aligned}
\prod_{\nu=1}^{\infty}\left(1-x^{a_{\nu}}\right)^{-1}= & \sum_{s=0}^{\infty} \frac{x^{s+d s(8-1) / 2}}{(1-x)\left(1-x^{2}\right) \cdots\left(1-x^{s}\right)} \\
= & \frac{1}{1-x}+\frac{x^{d+2}}{(1-x)\left(1-x^{2}\right)} \\
& +\frac{x^{3 d+8}}{(1-x)\left(1-x^{2}\right)\left(1-x^{3}\right)}+\cdots .
\end{aligned}
$$


Therefore $a_{1}=1$. Multiplying (7) by $1-x$ we have

$$
\prod_{\nu=2}^{\infty}\left(1-x^{a_{\nu}}\right)^{-1}=1+\frac{x^{d+2}}{1-x^{2}}+\frac{x^{3 d+3}}{\left(1-x^{2}\right)\left(1-x^{3}\right)}+\cdots .
$$

Hence $a_{2}=d+2$. Multiplying (8) by $\left(1-x^{d+2}\right)$ we have

$$
\begin{gathered}
\prod_{\nu=3}^{\infty}\left(1-x^{a_{\nu}}\right)^{-1}=1-x^{d+2}+\frac{x^{d+2}}{1-x^{2}}-\frac{x^{2 d+4}}{1-x^{2}} \\
+\frac{x^{3 d+3}}{\left(1-x^{2}\right)\left(1-x^{3}\right)}+\cdots
\end{gathered}
$$

If $d$ is odd the coefficient of $x^{2 d+4}$ on the right of (9) is seen to be -1 . This contradicts the fact that the product on the left, when expanded in powers of $x$, has non-negative coefficients. Hence $d$ must be even. In this case the right-hand member of (9) simplifies so that we have

$$
\begin{gathered}
\prod_{\nu=3}\left(1-x^{a_{\nu}}\right)^{-1}=1 \\
+x^{d+4}+x^{d+6}+\cdots+x^{2 d+2}+x^{3 d+3} \\
+x^{3 d+5}+x^{3 d+6}+\cdots .
\end{gathered}
$$

Hence $a_{3}=d+4$, and since $a_{\nu}+a_{\mu}>2 a_{3}=2 d+8$ we can conclude that $a_{4}=d+6, a_{5}=d+8, \cdots, a_{d / 2+2}=2 d+2$. Multiplying both sides of (10) by the corresponding factors we have

$$
\begin{aligned}
\prod_{\nu=3+d / 2}\left(1-x^{a_{\nu}}\right)^{-1}= & \prod_{\lambda=4}^{d+2}\left(1-x^{d+\lambda}\right)\left(1+x^{d+4}+x^{d+6}+\cdots\right. \\
& \left.+x^{2 d+2}+x^{3 d+3}+x^{3 d+5}+x^{3 d+6}+\cdots\right)
\end{aligned}
$$

We now consider the coefficient of $x^{2 d+8}$ on both sides of (11). Since $d$ is even, $3 d+3 \neq 2 d+8$ and $d \geqq 4$, so that $2 d+8<3 d+5$. Therefore the coefficient of $x^{2 d+8}$ on the right of (11) is -1 . As before this contradicts the fact that the coefficients on the left are non-negative. Hence we have the theorem.

THEOREM 3. The number $q_{d}(n)$ of partitions of $n$ into parts differing by $d$ or more is not equal to the number of partitions of $n$ into distinct parts taken from any set $S$ of integers whatsoever, except when $d=1$.

REMARKs. The theorem for $d=0$ is obvious since the first mentioned partitions are, in this case, unrestricted and hence more numerous than any other kind of partition. For $d=1$, the exception is of course necessary but trivial since in this case $S$ may be taken as the set of all integers. Hence we are interested in proving the theorem for $d \geqq 2$. 
Proof. Suppose the theorem to be false and let $S$ exist consisting of the integers $a_{1}<a_{2}<a_{3}<\ldots$. Then comparing the generating functions of the two kinds of partitions described in the theorem we have, by Theorem 1 ,

$$
\begin{aligned}
\prod_{\nu=1}\left(1+x^{a_{\nu}}\right)= & \frac{1}{1-x}+\frac{x^{d+2}}{(1-x)\left(1-x^{2}\right)} \\
& +\frac{x^{3 d+3}}{(1-x)\left(1-x^{2}\right)\left(1-x^{3}\right)}+\cdots .
\end{aligned}
$$

Therefore $a_{1}=1$. Multiplying both sides of (12) by $1-x$ we have

$$
\left(1-x^{2}\right) \prod_{\nu=2}\left(1+x^{a_{\nu}}\right)=1+\frac{x^{d+2}}{1-x^{2}}+\frac{x^{3 d+3}}{\left(1-x^{2}\right)\left(1-x^{3}\right)}+\cdots
$$

Let $\lambda$ be defined by

$$
2^{\lambda-1}<d+2 \leqq 2^{\lambda} .
$$

Since the coefficients of $x^{k}$ on the right of (13) vanish for $0<k<d+2$ we must have $a_{2}=2, a_{3}=2^{2}, a_{4}=2^{3}, \cdots, a_{\lambda}=2^{\lambda-1}$. Hence (13) may be written

$$
\left(1-x^{2^{\lambda}}\right) \prod_{\nu>\lambda}\left(1+x^{a_{\nu}}\right)=1+\frac{x^{d+2}}{1-x^{2}}+\frac{x^{3 d+3}}{\left(1-x^{2}\right)\left(1-x^{3}\right)}+\cdots
$$

We now separate two cases:

Case 1. $d$ is even. Let us compare the coefficient of $x^{2 \lambda}$ on both sides of (15). Since $3 d+3>2^{\lambda}$ by (14), the coefficient of $x^{2 \lambda}$ on the right of (15) is clearly +1 . Since the sum of any two $a$ 's occurring in (15) is greater than $2 a_{\lambda}=2^{\lambda}$, the coefficient of $x^{2 \lambda}$ on the left of (15) is either 0 or -1 , according as one of the $a_{\nu}$ has the value $2^{\lambda}$ or not. In either case a contradiction exists disposing of Case 1.

Case 2. $d$ is odd and greater than or equal to 3 . In this case the powers of $x$ occurring on the right of (15) are the odd powers from $x^{d+2}$ to $x^{3 d+2}$. Hence $x^{\lambda^{\lambda}}$ does not appear on the right. Since the sum of two $a$ 's on the left exceeds $2^{\lambda}$, one of these $a$ 's must be $2^{\lambda}$. Setting the corresponding factor $1+x^{2^{\lambda}}$ outside the product sign and developing the rest in powers of $x$ we have

$$
\begin{aligned}
\left(1-x^{2^{\lambda+1}}\right)\left(1+\sum_{\nu>\lambda} \sum_{a, \forall 2^{\lambda}} x^{a_{\nu}}+\sum_{\left.\mu>\nu>\lambda, a_{\mu \ngtr 2^{\lambda} \neq a_{\nu}} x^{a_{\nu}+a_{\mu}}+\cdots\right)}\right. \\
=1+\frac{x^{d+2}}{1-x^{2}}+\frac{x^{3 d+3}}{\left(1-x^{2}\right)\left(1-x^{3}\right)}+\cdots .
\end{aligned}
$$


Comparing the early powers of $x$ we see that $a_{\lambda+1}=d+2$, $a_{\lambda+2}=d+4, \cdots$, so that $a_{\nu}+a_{\mu} \geqq a_{\lambda+1}+a_{\lambda+2} \geqq 2 d+6$. Hence the $a$ 's in (16) are the successive odd numbers beginning with $d+2$ and extending at least as far as $2 d+5$ since this latter number is less than $2^{\lambda+1}$ on the one hand (in view of (14)) and $3 d+3$ on the other, since $d \geqq 3$. We now consider the coefficient of $x^{3 d+6}$ on both sides of (16). Since $3 d+6$ is odd, it is not the sum of two $a$ 's, since the $a$ 's are odd, nor is $3 d+6$ the sum of three or more $a$ 's, since such a sum would exceed $3 a_{\lambda+1}=3 d+6$. Hence any contribution to the coefficient of $x^{3 d+6}$ on the left of (16) must arise from the multiplication of $\left(1-x^{2^{\lambda+1}}\right)$ by $\sum x^{a_{v}}$ and so is either \pm 1 or 0 . However it is clear that the coeffcient of $x^{3 d+6}$ on the right of (16) is precisely 2. This contradiction proves the theorem.

University of CaLifornia 